\title{
Tilted-Lorentz symmetry
}

\author{
S A Jafari \\ Department of Physics, Sharif University of Technology, Tehran, Iran \\ E-mail: jafari@sharif.edu
}

(Received 24 March 2019 ; in final form 29 September 2019)

\begin{abstract}
Dirac cone can be tilted in condensed matter setting. As a result of tilt, the Lorentz symmetry is reduced to what we call tilted-Lorentz symmetry. In this paper, we derive the tilted-Lorentz transformations that leave a world with tilted Dirac cone invariant.
\end{abstract}

Keywords: tilted Lorentz group, spacetime in solid state physics

For full article, refer to the Persian section 